Recepción: 20 / 04 / 2017

Aceptación: 20 / 05 / 2017

Publicación: 15 / 07 / 2017
Ciencias económicas y empresariales Artículo Científico

\title{
Alimentación sistematizada vs Alimentación tradicional en la producción de camarón Vannamei
}

\author{
Systematic feeding vs Traditional feeding in \\ Vannamei shrimp production
}

\section{Sistematizada vs abastecimento alimentar tradicional na produção de camarão vannamei}

Michelle A. Varas-Chiquito ${ }^{\text {I }}$ michelle.varasch@ug.edu.ec

Leslie León-Bassantes ${ }^{\text {II }}$ leslie.leonb@ug.edu.ec

Ulises Villacis-Chancay ${ }^{\text {III }}$ ulises.villacisch@ug.edu.ec

César A. Alcívar-Aray ${ }^{\mathrm{IV}}$ cesar.alcivarar@ug.edu.ec

Correspondencia: michelle.varasch@ug.edu.ec 


\section{Resumen}

A nivel mundial Ecuador es reconocido como el país donde se viene practicando la acuicultura por ya más de 40 años, siendo el pionero en la industria camaronera en el continente americano con una mayor participación sobre las exportaciones totales de todos los países de América. Ecuador ha incrementado el volumen de producción en comparación a años anteriores en un 25\%, al pasar de 475 millones de libras a 540 millones de libras aproximadamente en lo que va del año; en cuanto a las exportaciones de camarón se representan en 2.500 millones de dólares. El presente análisis muestra el incremento de la producción a partir de la inclusión tecnológica y sistematización del proceso de alimentación que han adoptado algunas de las granjas más grandes del país para aumentar su producción camaronera. Los resultados que se presentan en el artículo es aplicando la metodología analítica documental donde se demuestra como los sistemas están permitiendo incrementar los niveles de producción camaronera a la vez que se expresan los resultados a través de comparaciones durante el proceso de análisis del crecimiento en mercados paralelos, revelando la eficiencia de los estudios realizados. Estos estudios permiten que el resultado y la metodología utilizados puedan ser usados en estudios similares para alcanzar mejores resultados en próximas investigaciones.

Palabras claves: Producción; alimentación; acuícola; tecnología; resultados. 


\section{Abstract}

Globally Ecuador is recognized as the country where aquaculture has been practiced for more than 40 years, being the pioneer in the shrimp industry in the American continent with a greater participation on the total exports of all the countries of America. Ecuador has increased the production volume compared to previous years by $25 \%$, from 475 million pounds to 540 approximately so far this year, while shrimp exports represent 2.5 billion dollars. The present analysis shows the increase in production from the technological inclusion and systematization of the feeding process that some of the largest farms in the country have adopted to increase their shrimp production. The results that will be shown in the present article will be following the analytical documentary methodology where it will be demonstrated how the systems are allowing to increase the levels of shrimp production, while the results will be made comparative during the process of analysis of the growth in parallel markets, showing the efficiency of the studies analyzed so that the results and the methodology will be used in similar studies.

Key words: Production; food, aquaculture; technology; results. 


\section{Resumo}

Worldwide Equador é reconhecido como o país onde a aquicultura tem sido praticada há mais de 40 anos, sendo o pioneiro na indústria do camarão nas Américas, com uma maior parcela do total das exportações de todos os países americanos. Equador tem aumentado o volume de produção em relação aos anos anteriores em 25\%, de 475 milhões de libras para 540 milhões de libras em cerca de agora este ano; Quanto às exportações de camarão são responsáveis por 2.500 milhões de dólares. Esta análise mostra o aumento da produção a partir da inclusão tecnológica e sistematização de processo de alimentação que adotaram algumas das maiores fazendas no país para aumentar a produção de camarão. Os resultados apresentados no artigo, aplicando o filme metodologia analítica que mostra tais sistemas estão permitindo o aumento dos níveis de produção de camarão, enquanto os resultados através de comparações são expressos durante a análise do crescimento em mercados paralelos revelando a eficiência dos estudos. Estes estudos permitem que os resultados e metodologia pode ser utilizado em estudos similares para alcançar melhores resultados em pesquisas futuras.

Palavras-chave: Produção; alimentos; aquicultura; tecnologia; resultados. 


\section{Introducción}

Tradicionalmente el negocio medular del sector camaronero consistía en el proceso de engorde o generación de biomasa de camarón a partir de dietas balanceadas con el uso de la infraestructura disponible, la misma se fundamentaba en un canoero que recorría toda la piscina vertiendo la dieta balanceada, esa dieta debe tener ciertas características, las cuales pueden ser clasificadas en dos grupos: Aspectos fisiológicos del sistema digestivo de los camarones: La ausencia de verdaderos dientes u órganos masticadores que les permiten a éstos manejar y digerir alimentos "gruesos" en cuanto al tamaño de las partículas; es decir, los camarones no requieren partículas de alimento demasiado finas. El proceso selectivo que hacen los camarones al tamaño de las partículas del alimento antes de pasar a la hepatopáncreas que es el sitio donde se realiza la verdadera digestión. Esta selectividad es una acción combinada de varios órganos del sistema digestivo que culmina en la cámara pilórica donde existe una especie de filtro que solo deja pasar a la hepatopáncreas, para la digestión, a aquellas partículas menores a un micrón; las que no cumplen este requisito retornan al molino gástrico a ser molidas otra vez.

Aspectos inherentes a la estabilidad física del alimento: El hábito alimenticio de los camarones de comer lentamente y de manipular los pellets o pelets hasta llevarlos a la boca ocasiona indudablemente las primeras pérdidas de nutrientes en el agua. La idea es que los pelets no se desintegren hasta que sean consumidos completamente por el camarón y resistan a la acción lixiviante del agua.

Por tanto, podemos decir que la alimentación es el elemento principal del costo de producción en la camaronicultura y debido a esto, es considerado el factor de mayor importancia económica en las diferentes especies de camarón que se cultivan en el mundo. Por otro lado, los trabajos científicos y 
técnicos relacionados con los métodos de alimentación tienen interés en los camaronicultores, además de preguntas como: ¿Cuándo alimentar? ¿Cómo alimentar? y ¿Cuánto alimento ofrecer? son cuestionamientos de cuya respuesta puede depender la diferencia entre obtener una ganancia importante o no. Si bien es cierto que actualmente existen métodos tradicionales de calcular la cantidad de alimento que se debe agregar a un estanque de producción, estos se consideran principalmente en la biomasa total, que se calcula basándose en biometrías periódicas de los organismos del estanque en cuestión. No obstante, aun y cuando este es un método que efectivamente nos permite conocer la cantidad de alimento a agregar diariamente (y que es un porcentaje determinado en base a la biomasa total), es necesario hacer notar que dentro de la población de camarones no todos se encuentran en las mismas condiciones fisiológicas a la vez, en otras palabras, es cuando la necesidad de tener mejoras en la alimentación y producción han conducido a los camaroneros a la sistematización de los procesos de alimentación de las larvas.

Ya hace 2 años aproximadamente, donde se opera la alimentación asistida por software, que consiste en un alimentador automático quien realiza el proceso varias veces al día pero de forma temporizada, de manera que la alimentación se hace dosificada basados en el sistemas acústicos de alimentación de camarones a través del ruido emitido por los crustáceos al momento de alimentarse, lo que se analiza a través de un dispositivo llamado hidrófono que envía los datos que permiten que los algoritmos del software de control alimenticio y monitoreo ambiental realicen el control del ritmo de alimentación y se informa al biólogo responsable de la camaronera a través de una PC o asistente digital personal, lo que permite adaptar el ritmo de alimentación en caso de requerirse. Este proceso sistematizado permite el incremento de la producción camaronera, ya en este proceso intervienen algunas variables como tener camarones más grandes en mayor tiempo y reducir significativamente el índice de mortandad de las larvas por contaminación de las piscinas. 
El camarón actualmente es el segundo producto no petrolero de mayor exportación después del banano.

Se considera que el buen momento del gremio ha derivado en mayor inversión para tecnificar los procesos al incluir la tecnología y la genética, lo que ha permitido un desarrollo sostenido del sector. Si bien es cierto el buen momento que atraviesa el camarón ecuatoriano, se instituye sobre dos sucesos importantes, el primero que los precios del crustáceo se han incrementado desde el año 2010 y el aumento de la producción; esto deriva de la inclusión de procesos sistemáticos y tecnológicos.

El sector camaronero ecuatoriano se encuentra en crecimiento en cuanto al volumen de su producción, según proyecciones de la Cámara Nacional de Acuacultura.

El Ecuador ha incrementado el volumen de producción en comparación a años anteriores en un 25\%, al pasar de 475 millones de libras a 540 millones de libras aproximadamente en lo que va del año, en cuanto a las exportaciones de camarón se representan en 2.500 millones de dólares y esto gracias a la inclusión de la tecnología en procesos como la alimentación y oxigenación de las piscinas.

\section{Importancia del tema}

Actualmente en Ecuador existen unas 210.000 hectáreas dedicadas al camarón, de estas el 60\% están en la provincia del Guayas, un 15\% en el Oro , un 9\% en Esmeraldas, otro 9\% en Manabí y el 7\% en la provincia de Sta. Elena, cabe destacar que existe un crecimiento profesional en el área de alimentación y de laboratorios porque hay mayor trabajo genético y mayor investigación sobre las 
larvas para producirlas más resistentes a enfermedades y por supuesto incrementar sus niveles de calidad.

Por su parte los productores trabajan en el reforzamiento de la infraestructura de las piscinas y la sistematización de procesos, así por ejemplo los muros de las piscinas, mejoramiento de las zonas de compuertas por donde ingresa y sale el agua que es captada desde el estuario.

El crecimiento de la producción responde a mejoras en los procesos como: uso de larvas de laboratorio, alimentación orgánica, buen manejo de suelo, sistematización de la alimentación, entre otros. Es así como se considera que los ingresos no solo se invierten en mejoramiento de infraestructura, sino también en tecnología y genética; al poder incluir la tecnología en el proceso de alimentación de las larvas; se debe de resaltar que buena parte de los recursos que ingresan al sector se destinan a mejoras tecnológicas, al trabajar en estanques de pre cría para comenzar el ciclo de producción con animales de mayor tamaño y resistencia, así por ejemplo el uso de sistemas conocidos como 'raceways', que son tanques donde se colocan a las larvas de camarón y permanecen hasta que estén más grandes y fuertes para sobrevivir en las piscinas, es decir, se usan para acelerar el crecimiento de las larvas y así elevar la productividad del camarón, esta inversión puede costar hasta USD 250.000, del 30\% al 40\% de las camaroneras de la costa del país los tienen, ya que hace unos cinco años eran solo un 10\%; de esta manera se puede mejorar la calidad de alimentación y cuidados en los procesos de producción de forma progresiva.

La alimentación de la larvas en las granjas sin alimentación sistematizada “de forma tradicional”, es aquella donde una persona se dedica dos horas diarias a alimentar a las larvas, rociando unos $300 \mathrm{~kg}$ de balanceado (dieta balanceada) en la piscina, aproximadamente $150 \mathrm{~kg}$, cada vez que se alimenta a 
las larvas, produciendo que el exceso de balanceado descomponga el agua de la piscina y cause enfermedades en los camarones.

Por lo tanto se puede decir que los factores de crecimiento económico sin duda son la innovación tecnológica y el emprendimiento en el sector camaronero.

La producción camaronera y las exportaciones, son determinantes en la economía nacional, ya que están directamente orientadas al crecimiento y desarrollo sostenido a largo plazo; en la actualidad tienen un peso importante en la actividad económica de los países. Entre los principales aspectos que le dan relevancia a las actividades de producción camaronera están: el incremento de divisas al país, crecimiento económico, innovación tecnología, presencia comercial internacional, entre otras. La conversión alimenticia es decir, la cantidad de masa que se gana versus la cantidad de alimento entregado, también mejora sustancialmente y eso se traduce en una disminución de costos al incluir tecnología basada en un software cuyo sistema funciona con paneles solares, además permite medir la temperatura y el oxígeno del agua, así mismo, el sistema cuenta con alimentadores automáticos; cada uno tiene una capacidad de carga de 190 a 200 kilos, dependiendo de la densidad del alimento, la comida es administrada con un motor que con sus revoluciones logra repartirlo en un área de 500 metros cuadrados aproximadamente.

\section{Materiales y métodos.}

Para el desarrollo de este análisis se aplicó la metodología de análisis documental, que permitió evaluar la información obtenida acerca del sector acuícola, específicamente el de producción camaronero y observar el comportamiento del proceso sistematizado frente al tradicional para conocer la evolución del mismo en el transcurso del tiempo. Permitiendo ubicar a Ecuador en el 
quinto lugar del mundo en la producción de camarón en cautiverio, lo que se traduce en competitividad.

\section{Resultados}

Guayas es la principal provincia productora de camarón en el país, durante el 2015 presentó un total de 138 mil hectáreas cultivadas, representando el $65.23 \%$ del total nacional, seguido por El Oro con el $19.05 \%$, en tercer lugar Manabí con el $8.77 \%$ y por último Esmeraldas con el $6.94 \%$, como se puede apreciar en el siguiente gráfico:

Tabla 1.- Producción de camarón en Ecuador por provincia

\begin{tabular}{|l|l|}
\hline \multicolumn{2}{|l|}{ Producción de camarón por provincia año 2015} \\
\hline Provincia & Producción \\
\hline Guayas & $65.23 \%$ \\
\hline Oro & $19.05 \%$ \\
\hline Manabí & $8.77 \%$ \\
\hline Esmeraldas & $6.94 \%$ \\
\hline
\end{tabular}

Gráfico 1: Producción de camarón en Ecuador por provincia.

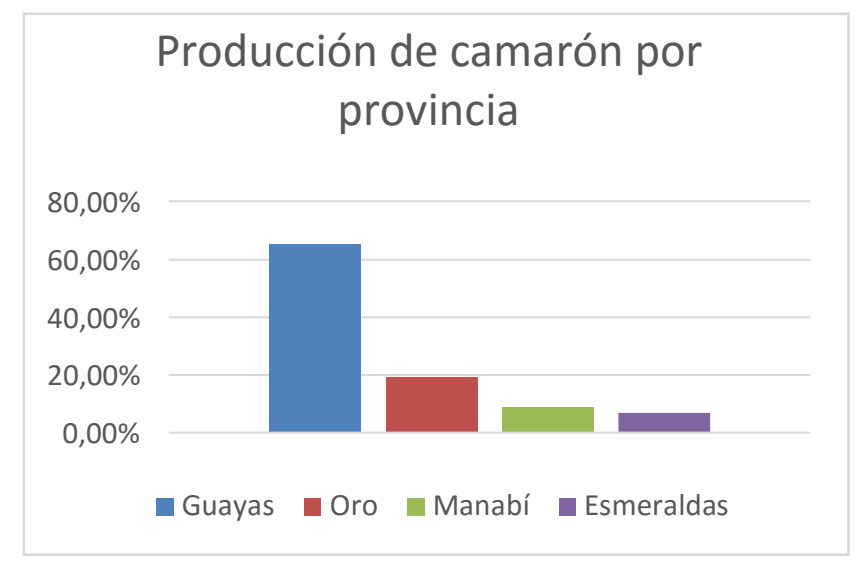


El gráfico 1, muestra los índices de producción camaronera por provincia, donde se evidencia que la provincia que genera mayor producción es Guayas, seguida de las provincias del Oro, Manabí y Esmeraldas respectivamente.

Tabla 2.- Comparación alimentación Tradicional Vs Sistematizada - indicador Nivel de Sobrevivencia.

\begin{tabular}{|c|c|c|}
\hline \multicolumn{3}{|c|}{ Alimentación Tradicional Vs Sistematizada } \\
\hline Indicador & $\begin{array}{c}\text { Método } \\
\text { Tradicional }\end{array}$ & $\begin{array}{c}\text { Método } \\
\text { Sistematizado }\end{array}$ \\
\hline $\begin{array}{c}\text { Nivel de } \\
\text { Sobrevivencia }\end{array}$ & 50 & 85 \\
\hline
\end{tabular}

Gráfico 2: Alimentación Tradicional vs Sistematizada - Nivel de sobrevivencia.

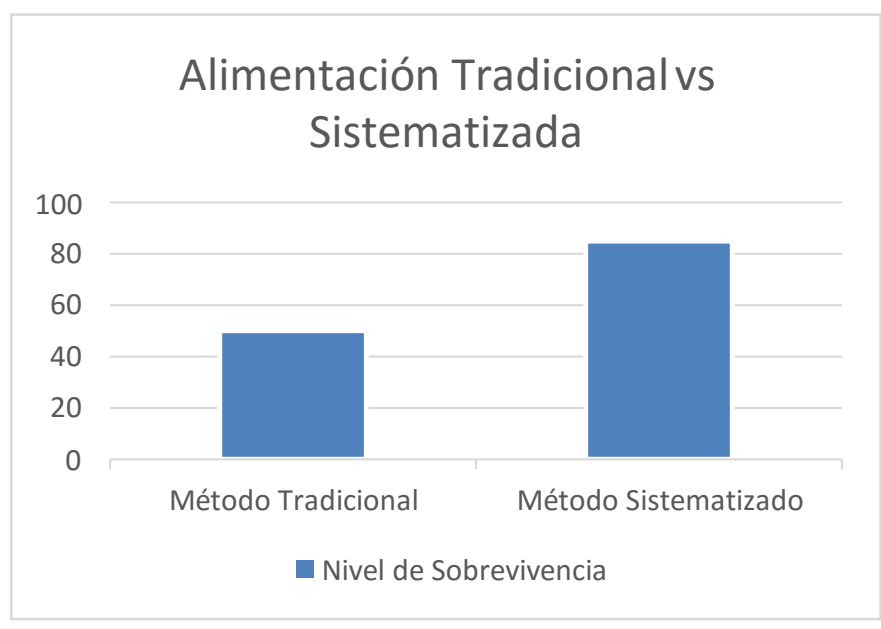

El gráfico 2, corresponde a la comparación de la alimentación tradicional vs la sistematizada, en el cual el indicador evaluado es el nivel de sobrevivencia, se puede observar que en las granjas donde se encuentra sistematizada la alimentación, el índice de sobrevivencia es mayor que en el método tradicional. 
Tabla 3.- Comparación alimentación Tradicional Vs Sistematizada - Indicador Factor de conversión.

\begin{tabular}{|c|l|l|}
\hline \multicolumn{3}{|c|}{ Alimentación Tradicional Vs Sistematizada } \\
\hline Indicador & Método Tradicional & Método Sistematizado \\
\hline Factor de conversión FCR & 1,2 & 2 \\
\hline
\end{tabular}

Gráfico 3: Alimentación Tradicional vs Sistematizada - Índice factor de conversión.

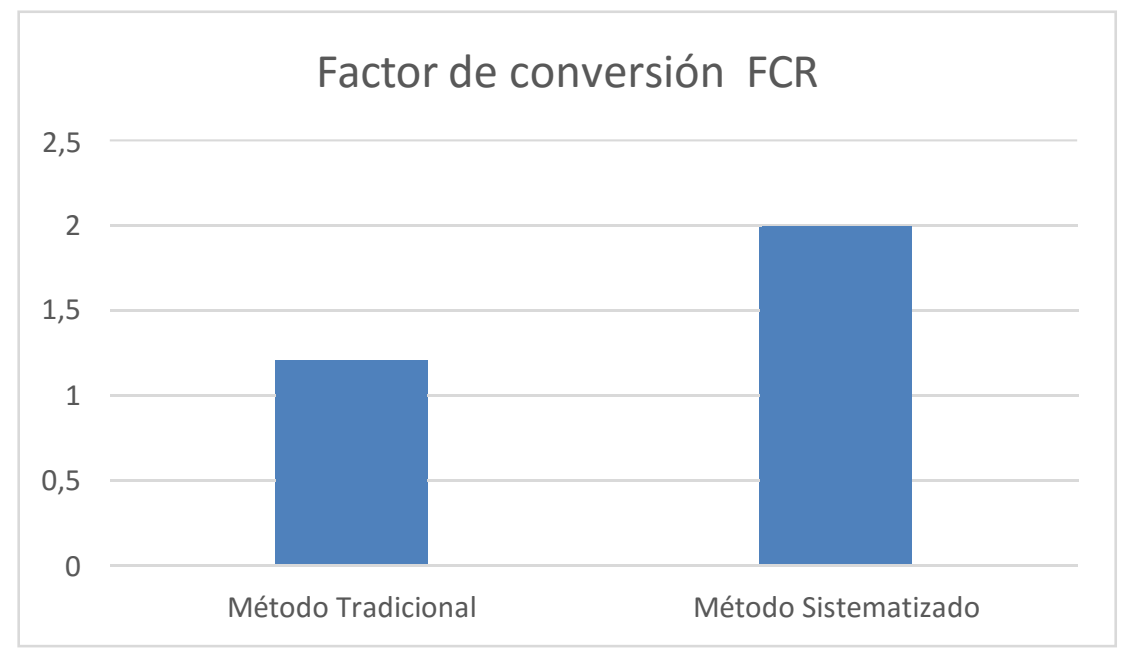

El gráfico 3, corresponde a la comparación de la alimentación tradicional vs la sistematizada, en el cual el indicador evaluado es el factor de conversión FCR que es por cada libra de balanceado cuantas libras de camarón se han obtenido. 
Tabla 4.- Comparación alimentación Tradicional Vs Sistematizada - Indicador libras de producción por hectárea de piscina.

\begin{tabular}{|l|c|c|}
\hline \multicolumn{3}{|c|}{ Alimentación Tradicional Vs Sistematizada } \\
\hline Indicador & Método Tradicional & Método Sistematizado \\
\hline $\mathrm{Lb} / \mathrm{Ha}$ & 2800 & 4500 \\
\hline
\end{tabular}

Gráfico 4: Alimentación Tradicional vs Sistematizada - Libras cosechadas por hectárea de piscina.

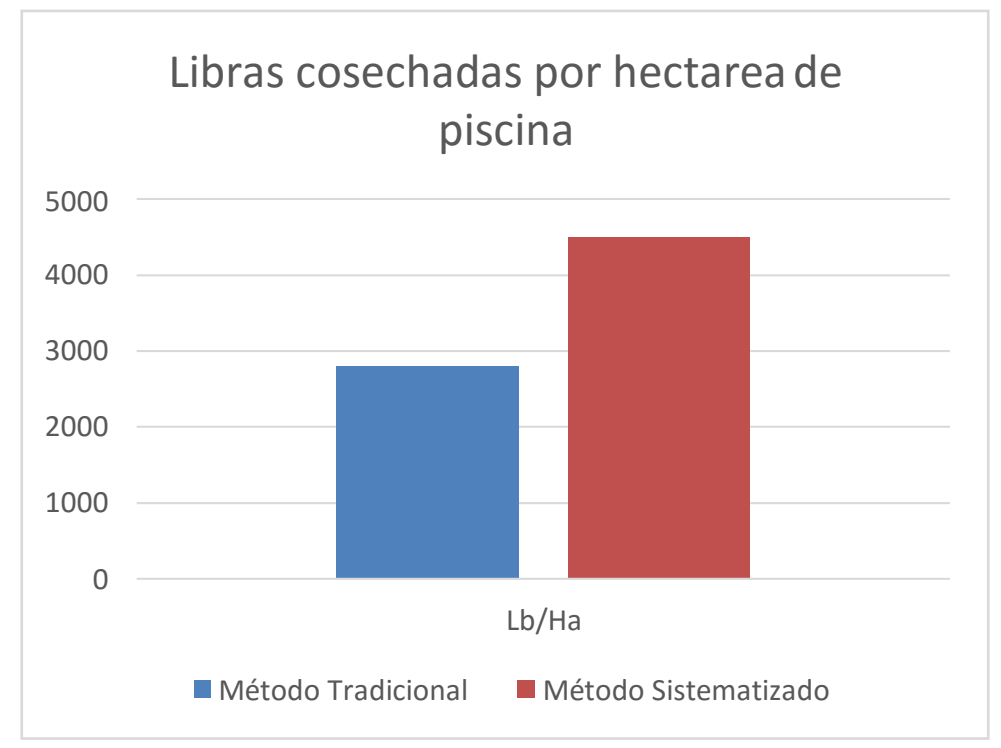

El gráfico 4, evidencia que las libras cosechadas en las camaroneras que cuentan con el sistema de alimentación son más productivas que aquellas donde se aplican métodos tradicionales de alimentación. 
Tabla 5.- Comparación alimentación Tradicional Vs Sistematizada - Índice Días de Cultivo.

\begin{tabular}{|c|l|l|}
\hline \multicolumn{3}{|c|}{ Alimentación Tradicional Vs Sistematizada } \\
\hline Indicador & Método Tradicional & Método Sistematizado \\
\hline Días de cultivo & 120 & 75 \\
\hline
\end{tabular}

Gráfico 5: Alimentación Tradicional vs Sistematizada - Indicador días de cultivo.

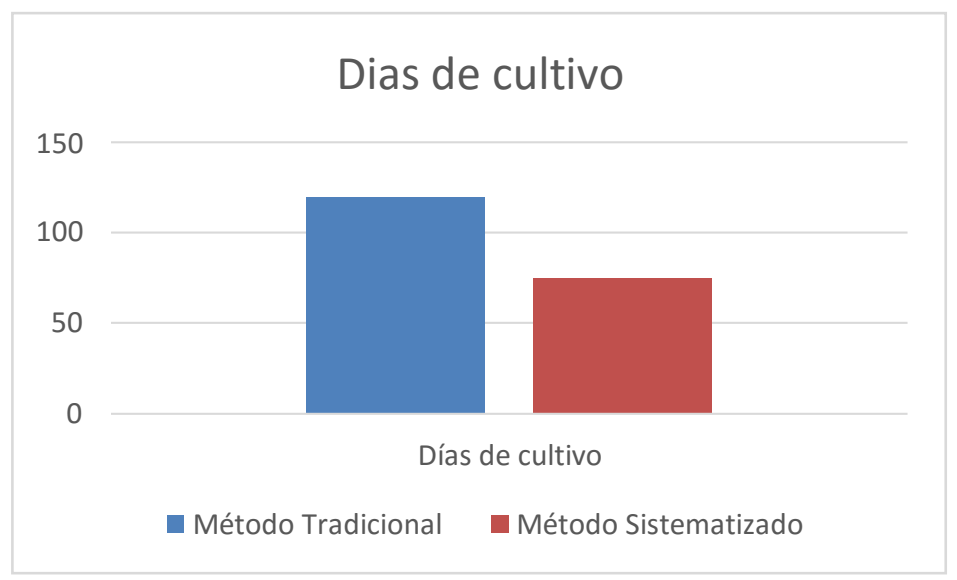

El gráfico 5, los días que se emplean para realizar el proceso de cultivo de los camarones por método de cultivo.

Tabla 6.- Comparación alimentación Tradicional Vs Sistematizada - Indicador Peso del camarón.

\begin{tabular}{|c|l|l|}
\hline \multicolumn{3}{|c|}{ Alimentación Tradicional Vs Sistematizada } \\
\hline Indicador & Método Tradicional & Método Sistematizado \\
\hline Peso camarón & 16 & 16 \\
\hline
\end{tabular}


Gráfico 6: Alimentación Tradicional vs Sistematizada - Indicador días de cultivo.

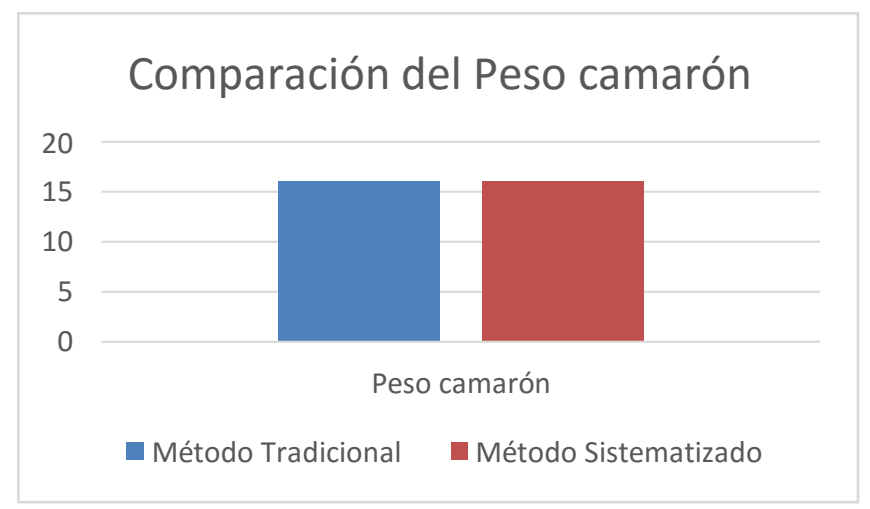

El gráfico 6, evidencia que los camarones logran alcanzar el peso deseado en relación a los días de cultivo.

Tabla 7.- Comparación alimentación Tradicional Vs Sistematizada - Indicador dosis diarias de alimentación.

\begin{tabular}{|c|l|l|}
\hline \multicolumn{3}{|c|}{ Alimentación Tradicional Vs Sistematizada } \\
\hline Indicador & Método Tradicional & Método Sistematizado \\
\hline Dosis diarias & 2 & 600 \\
\hline
\end{tabular}

Gráfico 7: Alimentación Tradicional vs Sistematizada - Indicador dosis diarias de alimentación.

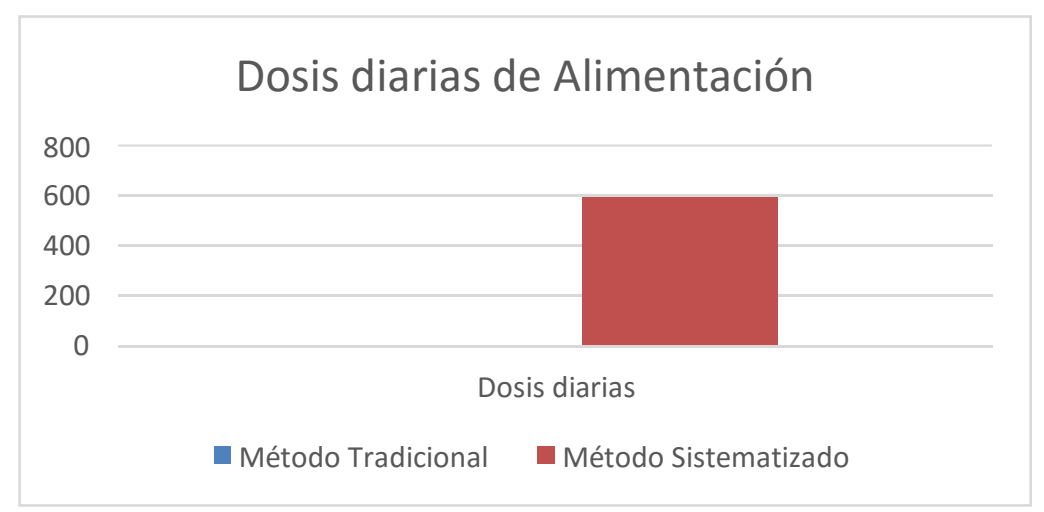


El gráfico 7, evidencia que con la alimentación sistematizada las dosis diarias de alimentación de los camarones es mucho mayor que la tradicional pero este proceso se realiza siempre que los camarones necesiten alimentarse evitando el desperdicio del balanceado y contaminación de las piscinas.

\section{Discusión}

La tecnificación y sistematización en el proceso de alimentación de los camarones está logrando incrementar significativamente sus niveles de producción, logrando tener camarones con mayor tamaño y peso en menor tiempo, además de una disminución en el índice de mortandad y disminución en la contaminación de las piscinas.

Es necesario destacar que dentro de la sistematización de las granjas camaroneras los procesos que se han tecnificados a través del uso de softwares que controlan, dosifican y regulan la alimentación de los camarones permiten generar reportes para los usuarios que son necesarios para establecer medidas que ayuden a incrementar la producción, además de contar con sistemas sónicos y sensores que permiten tener mayor control de la alimentación del crustáceo y de factores climáticos también.

La inclusión de la tecnificación y sistematización en las camaroneras se ha realizado mayormente en las granjas de aquellos productores camaroneros de mayores recursos económicos, quienes han visto a la tecnología como su mejor aliada al momento de incrementar la producción, incentivándolos a invertir en la misma, trayendo como beneficios: tener un aumento en la producción camaronera, es decir, los productores ecuatorianos podrán mantenerse en el sector a nivel mundial logrando competir contra los precios que establecen los asiáticos en el mercado, así mismo, se puede evidenciar que a medida que se tecnifiquen y sistematicen las camaroneras, para los pequeños productores será mucho más difícil poder sobrevivir en el mercado puesto que no podrán mejorar 
sus niveles de producción, también se comprobó que existe un incremento en la producción en relación al tamaño de la piscina, por lo que se ha elevado ese indicador. La cantidad de dosis de alimentación con un sistema tradicional se encuentra muy por debajo de la realizada en las granjas que poseen sistemas de alimentación tecnificados, por tanto, los camarones se alimentan cada vez que tengan hambre y no existe un desperdicio del alimento, permitiendo esto una disminución en los costos de producción y una disminución en la tasa de mortandad.

\section{Conclusiones}

- Las acciones de trabajo individual, autónomas empresarial, esfuerzo, inversión han permitido el incremento de la producción eficiente, logrando camarones más grandes en menor tiempo.

- $\quad$ En este análisis se determinaron grandes diferencias que van desde un aumento del $25 \%$ en promedio general, en todos los comparativos se ha evidenciado un incremento en un $62,22 \%$ de la producción y una disminución del $45 \%$ menos del tiempo de cosecha.

- Se ha demostrado que existen mejoras al poder ingresar a mercados que eran inaccesibles, luego de 14 años de la crisis de la mancha blanca, es así que hay especias como "Vanamei” que tienen mejor acceso a mercados de los países europeos y asiáticos, de esta forma se puede decir que el $27 \%$ del camarón exportado por Asia es ecuatoriano, del mismo modo el $33 \%$ de las exportaciones de los camarones "Vanamei" entran al mercado europeo a países como Italia y España.

\section{Bibliografía}

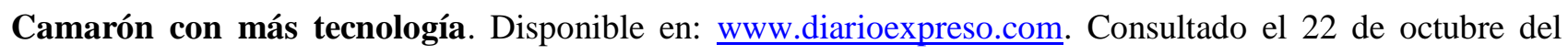
2016. 
Cámara nacional de acuacultura. Disponible en: www.cna-ecuador.com. Consultado el 17 de marzo del 2016.

Cámara nacional de acuacultura. Disponible en: www.cna-ecuador.com. Consultado el 17 de marzo del 2016.

Daniel, Meyer (2004). Introducción a la Acuacultura. Escuela Agrícola Panamericana Zamorano, Honduras.

Martínez (2002). Camarón y cultura Avances y Tendencias.

Morales (2010). Enfermedades del Camarón Detección mediante análisis en fresco e histopatología.

Páez, Osuna (2011). Metales en camarón de cultivo y silvestres. Disponible en: http://www.industriaacuicola.com/libros.htm. Consultado el 08 de octubre de 2016.

Prochure SF200. Disponible en: http://www.Apracom-ec.com. Consultado el 09 de octubre del 2016.

Pro Ecuador Instituto de promoción de exportaciones e inversión. Disponible en: www.proecuador.gob.ec. Consultado el 08 de octubre del 2016.

Revista Lideres. Disponible en: www.revistalideres.ec. Consultado el 11 de septiembre del 2016. 